\title{
Actividades de prevención primaria del pie diabético y actividades de autocuidado en pacientes con diabetes tipo 2 en el Hospital II Clínica Geriátrica San Isidro Labrador, Essalud
}

\author{
Primary prevention activities of the diabetic foot and self-care activities in patients \\ with type 2 diabetes in the Hospital II Clínica Geriátrica San Isidro Labrador, Essalud
}

Gloria Rosa Mesares Damiano ${ }^{\star \star}$, Keila Ester Miranda Limachi ${ }^{2}$

\section{RESUMEN}

Objetivos: determinar qué actividades de prevención primaria de pie diabético realiza el enfermero y qué actividades de autocuidado realiza el paciente con diabetes tipo2 en el servicio de Medicina del Hospital II Clínica Geriátrica San Isidro Labrador, Es Salud. Materiales y Métodos: descriptiva y transversal. Para la recolección de datos se aplicó dos cuestionarios. Resultados: Los resultados evidencian que el 83,6\% de enfermeros realiza la valoración de los pies, el 90,9\% imparte educación. El $76,4 \%$ pregunta si presentan cambios en la sensibilidad del pie y el $85,2 \%$ pregunta durante la entrevista si hay presencia de dolor en el pie, El 72,7\% brinda técnicas de autocuidado del pie, el $56,4 \%$ clasifica a sus pacientes en niveles de bajo o alto riesgo de desarrollar úlceras o amputaciones y el. 53,8\% informa al paciente sobre su categoría de riesgo de presentar pie diabético. El 92,7\% recomienda dejar de fumar y el $98,2 \%$ informa la importancia de asistir a su control. Con respecto a las prácticas de autocuidado que realizan los pacientes, el $46,4 \%$ presenta un nivel de autocuidado moderado, el $69,6 \%$ revisa sus pies, el $42,9 \%$ no revisa el interior de su calzado, el $60 \%$ no aplica cremas hidratantes, el 44,6 \% continúa agregando azúcar a sus bebidas, el $45,5 \%$ de los pacientes tiene sobrepeso, incluyendo ambos sexos. Conclusiones: la mayoría de los enfermeros, realiza bastantes actividades de prevención primaria del pie diabético. Por otro lado, existe una moderada práctica de autocuidado realizado por el paciente.

Palabras clave: Diabetes, prevención, enfermería, autocuidados, pie diabético. (Fuente: DeCS BIREME)

\section{ABSTRACT}

Objetives: To identify which primary prevention of diabetic foot hits the nurse and what self-care activities performed by the patient with type 2 diabetes. Materials and Methods: The type of study is descriptive and cross-sectional data collection survey was applied to nurses and diabetic patients over 18 years. Results: The results show that $83.6 \%$ of nurses performed the assessment of the foot, provides education 90.9\%. $72.7 \%$ provide foot self-care techniques. Recommended $92.7 \%$ quit smoking and $98.2 \%$ reported the importance of attending to his control. With regard to self-care practices performed by the patients, $46.4 \%$ have a moderate level of self-care, $69.6 \%$ check their feet, $42.9 \%$ did not check the inside of their shoes, $60 \%$ no apply moisturizers, $44.6 \%$ continues to add sugar to their drinks, $45.5 \%$ of overweight patients, including both sexes. Conclusions: Most of nurses take almost all activities of primary prevention of diabetic foot highlighting an important activity assessment of the foot. Furthermore, there is a regular practice of self-performed by the patient, especially in the daily observation of the foot.

Keywords: Diabetes, prevention, first aid, self-care, diabetic foot. (Source: MeSH NLM)

${ }^{1}$ Enfermera asistencial, Madrid España.

${ }^{2}$ Doctora en Ciencias de Enfermería, Universidad Peruana Unión, Lima, Perú. 


\section{INTRODUCCIÓN}

La diabetes tipo 2 representa el 95\% o más del total de casos en el mundo, siendo el tipo más común de diabetes que por lo general, ocurre en los adultos, pero cada vez se observa en niños, adolescentes y jóvenes adultos con sobrepeso. Está afectando especialmente a las personas en edad reproductiva. (Organización Mundial de la Salud, 2012). Se caracteriza por la resistencia a la insulina y una deficiencia relativa de dicha hormona, esta puede permanecer sin ser detectada, es decir, ser asintomática, durante muchos años y el diagnóstico suele producirse a partir de complicaciones asociadas o incidentalmente mediante un análisis de sangre $u$ orina que arroje resultados anormales. (Federación Internacional de la Diabetes, 2013). Una diabetes tipo2 no diagnosticada o mal controlada puede provocar la amputación de las extremidades inferiores, ceguera y enfermedad renal.

Asimismo, contribuye notablemente a la enfermedad cardíaca y al derrame cerebral, encontrándose entre las diez causas principales de discapacidad en el mundo (Federación Internacional de Diabetes 2011). Por otro lado, es importante recordar que el diagnóstico de diabetes es sinónimo de "Pie en riesgo". (Rebaza, 2011)

El Ministerio de Salud (2015), declara que la DM2 es una enfermedad que afecta a casi 2 millones de personas y es la décimo segunda causa de Años de Vida Saludable Perdidos (AVISA) y discapacidad en nuestra población, con tendencia creciente.

Solo en Lima, en el año 2015 se presentaron más de 50 mil casos nuevos de ese mal en hombres y mujeres de 39 a 50 años y 203 casos en niños de 0 a 17 años,

(Seclén, 2011/2012). Ante esta cruda realidad estadística de comorbilidad se ha realizado este trabajo de investigación para determinar qué actividades de prevención primaria de pie diabético realiza la enfermera y qué actividades de autocuidado realiza el paciente con diabetes tipo2, en el Hospital II Geriátrico San Isidro Labrador.

\section{MATERIAL Y MÉTODOS}

Esta investigación es de tipo cuantitativo, descriptivo y transversal. Para la recolección de datos se utilizó un instrumento que fue elaborado por Pinilla R., Barrera P, Rodríguez M., Rubio C, y Devia D. (2010), el cual ha sido modificado a la necesidad y características de la muestra de estudio, aplicado a 55 enfermeras(os) que están a cargo de pacientes con diabetes tipo 2 y con un año de experiencia laborando en el servicio de medicina del Hospital San Isidro Labrador y a 56 pacientes con diabetes tipo2, cognitivamente bien, mayores de edad, que no presenten pie diabético y se encuentran hospitalizados. Se verifico las propiedades psicométricas de los dos cuestionarios para lo cual los ítems fueron evaluados por expertos y la fiabilidad global del cuestionario y sus dimensiones se valoró calculando el índice de consistencia interna mediante el coeficiente Alpha de Cronbach, donde la fiabilidad global fue de .612 (instrumento de enfermeras) y .641 (instrumento del paciente). La aplicación de la encuesta se ejecutó por la investigadora, previa coordinación con los representantes de la institución, se les explicó a todas las enfermeras y pacientes el propósito de la investigación, siendo todo de carácter confidencial y anónimo, el tiempo de la ejecución de la encuesta duro 5 a 10 minutos, los datos fueron llevados a un análisis estadístico y confrontado con la teoría y otros estudios similares.

\section{RESULTADOS}

Se aprecia, en la Figura 1, que el $94,5 \%$ de los enfermeros realiza la mayoría de las actividades de prevención primaria del pie diabético y el $5,5 \%$ realiza pocas actividades de prevención primaria del pie diabético.

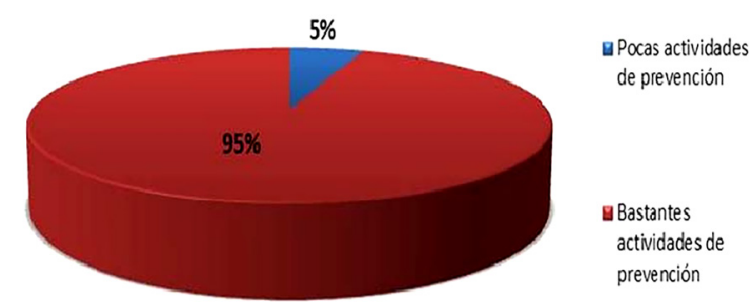

Figura 1. Actividades primarias que realizan los enfermeros para la prevención del pie diabético. 
Actividades de prevención primaria del pie diabético y actividades de autocuidado en pacientes con diabetes tipo 2 en el Hospital II Clínica Geriátrica San Isidro Labrador, Essalud

En la tabla 1 se observa con respecto a la pregunta si realiza la valoración respectiva a los pies de sus pacientes, el $83,6 \%$ de los enfermeros realiza dicha actividad, el 90,9\% imparte educación; no obstante, el $56,4 \%$ no realiza la inspección tanto por dentro como por fuera del calzado y calcetines. El $76,4 \%$ pregunta si presenta cambios en la sensibilidad y el $85,2 \%$ también pregunta durante la entrevista si hay presencia de dolor en el pie. Sin embargo, el 56,4\% de enfermeros clasifica a sus pacientes en niveles de bajo o alto riesgo de desarrollar úlceras o amputaciones, y el 53,8\% informa al paciente sobre su categoría de riesgo de presentar pie diabético. De acuerdo a la pregunta si enseñan técnicas de autocuidado del pie, un $72,7 \%$ sí realiza técnicas de autocuidado, el $92,7 \%$ recomienda dejar de fumar y la gran mayoría de los enfermeros $(98,2 \%)$ informa a sus pacientes la importancia de asistir a su control.

Tabla 1

Actividades de prevención primaria del pie diabético realizadas por las enfermeras.

\begin{tabular}{|c|c|c|c|c|c|}
\hline \multirow[t]{3}{*}{$\mathbf{N}$} & \multirow[t]{3}{*}{ Ítems } & \multicolumn{4}{|c|}{ Nivel de autocuidado } \\
\hline & & \multicolumn{2}{|r|}{ No } & \multicolumn{2}{|r|}{ Si } \\
\hline & & $\mathbf{N}$ & $\%$ & $\mathbf{N}$ & $\%$ \\
\hline P1 & ¿Realiza valoración de los pies a los pacientes con diabetes? & 9 & $16,4 \%$ & 46 & $83,6 \%$ \\
\hline $\mathrm{P} 2$ & $\begin{array}{l}\text { ¿Realiza educación sobre el cuidado del pie a las personas con } \\
\text { diabetes? }\end{array}$ & 5 & $9,1 \%$ & 50 & $90,9 \%$ \\
\hline P3 & $\begin{array}{l}\text { ¿Inspecciona el tipo de zapatos y calcetines por dentro y por } \\
\text { fuera? }\end{array}$ & 31 & $56,4 \%$ & 24 & $43,6 \%$ \\
\hline P5 & $\begin{array}{l}\text { ¿Alguna vez le ha preguntado a su paciente si presenta cambios } \\
\text { en la sensibilidad de los pies? }\end{array}$ & 13 & $23,6 \%$ & 42 & $76,4 \%$ \\
\hline P6 & $\begin{array}{l}\text { ¿Alguna vez le ha preguntado a su paciente si presenta dolor en } \\
\text { las extremidades inferiores? }\end{array}$ & 8 & $14,8 \%$ & 46 & $85,2 \%$ \\
\hline P7 & $\begin{array}{l}\text { ¿Clasifica a los pacientes en pie de "bajo" o "alto" riesgo de } \\
\text { desarrollar úlceras/amputaciones? }\end{array}$ & 24 & $43,6 \%$ & 31 & $56,4 \%$ \\
\hline P8 & $\begin{array}{l}\text { En caso afirmativo, ¿informa al paciente sobre su categoría de } \\
\text { riesgo? }\end{array}$ & 24 & $46,2 \%$ & 28 & $53,8 \%$ \\
\hline P10 & ¿Enseña técnicas de autocuidado del pie? & 15 & 27,35 & 40 & $72,7 \%$ \\
\hline P11 & $\begin{array}{l}\text { ¿Si tiene un paciente que fuma y tiene diabetes, le ha } \\
\text { recomendado que deje de fumar? }\end{array}$ & 4 & $7,3 \%$ & 51 & $92,7 \%$ \\
\hline $\mathrm{P} 12$ & $\begin{array}{l}\text { ¿Informa la importancia de la asistencia al hospital para el control } \\
\text { de la diabetes de sus pacientes? }\end{array}$ & 1 & $1,8 \%$ & 54 & $98,2 \%$ \\
\hline
\end{tabular}

La figura 2 permite apreciar que el $42,6 \%$ de enfermeros imparte todas las técnicas de autocuidado para la prevención del pie diabético como: (a) cuidado de las uñas (b) lavado, secado y uso de cremas para el pie (c) revisión diaria del pie y (d) el tipo de zapatos recomendados para su actividad diaria, uso de medias y no caminar descalzo. También se observa que el $21,3 \%$ de los enfermeros imparte temas del autocuidado obviando la revisión diaria del pie. 


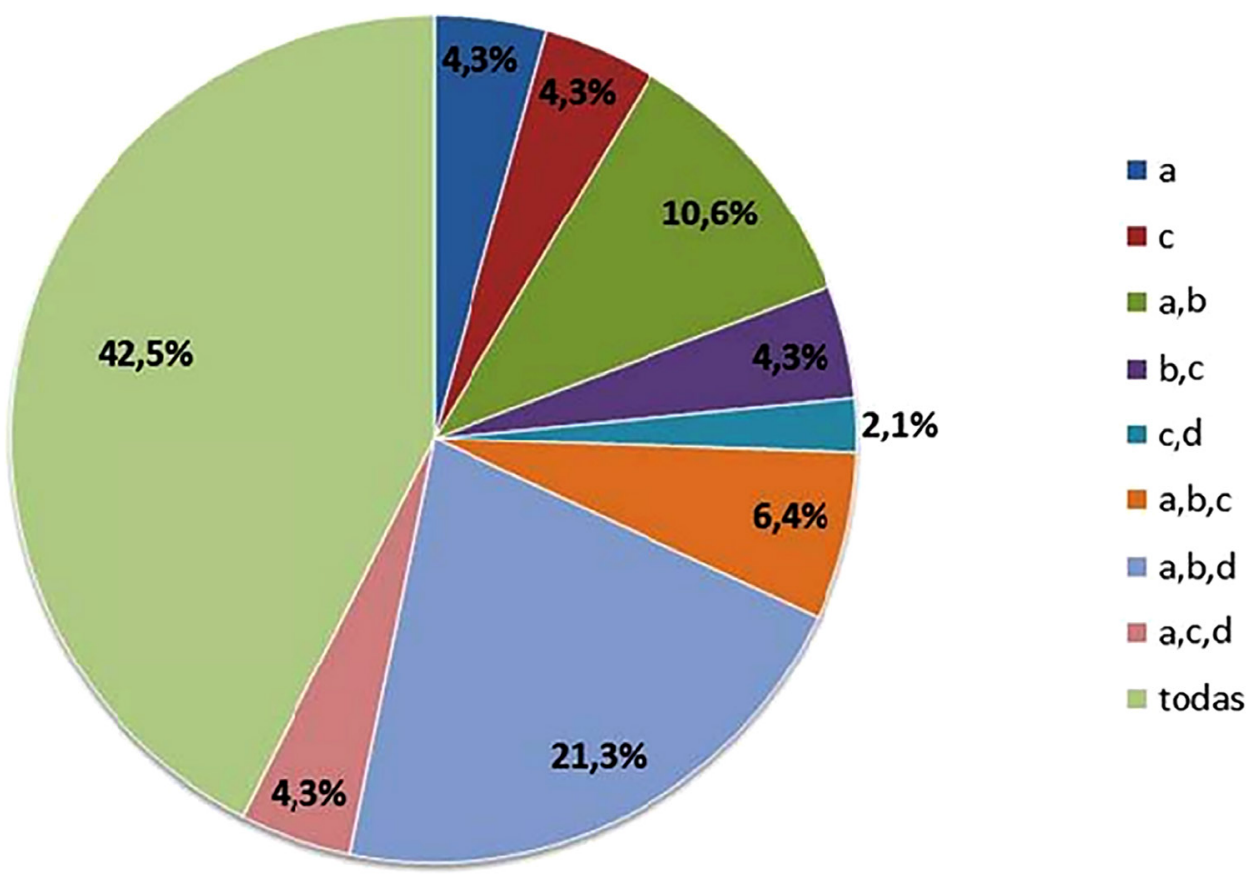

Figura 2. Técnicas de autocuidado impartidos por los enfermeros: (a) cuidado de las uñas, (b) lavado, secado y uso de cremas para el pie, (c) revisión diaria del pie, (d) tipo de zapato recomendado para su actividad diaria, uso de medias y no caminar descalzo en casa.

En la tabla 2 muestra que el $46,4 \%$ de los pacientes presenta un nivel de autocuidado moderado de sus pies, el $30,4 \%$ mantiene un nivel de autocuidado alto, y el $23.2 \%$, posee un nivel de autocuidado bajo.

Tabla 2

Nivel de autocuidado realizado por el paciente para prevención del pie diabético.

\begin{tabular}{lcc} 
Nivel de Autocuidado & N & \\
\hline Bajo & 13 & 23,2 \\
Moderado & 26 & 46,4 \\
Alto & 17 & 30,4 \\
Total & 56 & 100 \\
\hline
\end{tabular}

La tabla 3 permite apreciar que el $69,6 \%$ de pacientes revisa su pie todos los días, el $78,6 \%$ no considera importante elegir el color de los calcetines al momento de comprarlos, el $42,9 \%$ no revisa el interior del calzado y el $60 \%$ no aplica cremas o vaselina a su pie. Sin embargo, que el $87,5 \%$ sí seca los espacios interdigitales del pie, el $91,1 \%$ no camina descalzo en su casa u otros lugares. El $30,4 \%$ no considera importante la temperatura del agua antes de asearse el pie, el $87,5 \%$ evita el uso de objetos (compresas, botellas, mantas eléctricas) para calentar sus pies. Con respecto los ítems 12 , el $83,9 \%$ respondió que sí comunicaría inmediatamente a su médico tratante sobre alguna alteración producida en sus pies, el 30,4\% tiene preferencia por consumir alimentos fritos, el $69,6 \%$ continúan consumiendo una dieta compuesta por carnes, pescado y huevos diariamente, el $57,1 \%$ de los pacientes diabéticos consume más de 2 carbohidratos (harinas) durante el día y el 44,6\% continúan agregando azúcar a sus bebidas. 
Actividades de prevención primaria del pie diabético y actividades de autocuidado en pacientes con diabetes tipo 2 en el Hospital II Clínica Geriátrica San Isidro Labrador, Essalud

Tabla 3

Actividades de autocuidado realizados por el paciente para prevenir el pie diabético.

\begin{tabular}{|c|c|c|c|c|c|}
\hline \multirow[t]{3}{*}{$\mathrm{N}$} & \multirow[t]{3}{*}{ Ítems } & \multicolumn{4}{|c|}{ Nivel de autocuidado } \\
\hline & & \multicolumn{2}{|c|}{ No } & \multicolumn{2}{|l|}{$\mathrm{Si}$} \\
\hline & & $\mathrm{N}$ & $\%$ & $\mathrm{~N}$ & $\%$ \\
\hline P1 & $\begin{array}{l}\text { ¿Revisa la presencia de lesiones en los pies todos los } \\
\text { días? }\end{array}$ & 17 & $30.4 \%$ & 39 & $69.6 \%$ \\
\hline P2 & $\begin{array}{l}\text { ¿Tiene en cuenta el color a la hora de comprar las } \\
\text { medias? }\end{array}$ & 44 & $78.6 \%$ & 12 & $21.4 \%$ \\
\hline P3 & $\begin{array}{l}\text { ¿Revisa el interior del zapato siempre antes de } \\
\text { introducir su pie? }\end{array}$ & 24 & $42.9 \%$ & 32 & $57.1 \%$ \\
\hline P4 & ¿Aplica cremas hidratante a diario en los pies? & 33 & $60 \%$ & 22 & $40 \%$ \\
\hline P5 & $\begin{array}{l}\text { ¿Seca espacios entre los dedos del pie después del } \\
\text { baño, todos los días? }\end{array}$ & 7 & $12.5 \%$ & 49 & $87.5 \%$ \\
\hline P6 & ¿Camina descalzo en casa u otros lugares? & 51 & $91.1 \%$ & 5 & $8.9 \%$ \\
\hline P7 & $\begin{array}{l}\text { ¿Tiene en cuenta la temperatura del agua a la hora } \\
\text { de lavarse los pies? }\end{array}$ & 17 & $30.4 \%$ & 39 & $69.6 \%$ \\
\hline P8 & $\begin{array}{l}\text { ¿Utiliza objetos calientes para calentar sus pies } \\
\text { (bolsas, compresas, botellas)? }\end{array}$ & 49 & $87.5 \%$ & 7 & $12.5 \%$ \\
\hline P9 & $\begin{array}{l}\text { ¿Consultaría inmediatamente a su médico si encuentra } \\
\text { algún problema en sus pies? }\end{array}$ & 9 & $16.1 \%$ & 47 & $83.9 \%$ \\
\hline P10 & ¿Prefiere consumir los alimentos fritos? & 39 & $69.6 \%$ & 17 & $30.4 \%$ \\
\hline P11 & ¿Consume carne, pescado, pollo o huevo diariamente? & 17 & $30.4 \%$ & 39 & $69.6 \%$ \\
\hline P12 & $\begin{array}{l}\text { ¿Consume más de dos carbohidratos (harinas) al día en } \\
\text { desayuno, almuerzo o cena? }\end{array}$ & 24 & $42.9 \%$ & 32 & $57.1 \%$ \\
\hline P13 & $\begin{array}{l}\text { ¿Adiciona azúcar a las preparaciones o consume } \\
\text { alimentos que contienen estos endulzantes? }\end{array}$ & 31 & $55.4 \%$ & 25 & $44.6 \%$ \\
\hline
\end{tabular}

\section{DISCUSIÓN}

Al analizar los resultados obtenidos en la investigación se encontró, en las características socio demográficas de la muestra, que casi la mitad de las enfermeros tienen entre 25 y 35 años de edad, la mayoría son mujeres y poseen de 1 a 5 años de ejercicio profesional en el área asistencial del Hospital II Geriátrico San Isidro Labrador, y la mayoría de los pacientes tienen de 61 a 80 años y una minoría es adulta entre 41 y 50 años, al respecto, Pinilla, Sánchez, Mejía (2011), señala que la mitad de su muestra fueron adulto de 30 a 64 años; más de la mitad son mujeres con grado de instrucción primaria, resultados parecidos se refleja en la investigación de Cárdenas y Flores (2010), donde señala que más de la mitad de su muestra fueron mujeres con escolaridad baja.

Con respecto a las actividades de prevención que realiza las enfermeras, es interesante señalar que la mayoría realiza la valoración del pie de forma general e imparte educación a los pacientes durante el tiempo de su hospitalización, siendo estos hallazgos una evidencia que se vienen ejecutando actividades de prevención del pie diabético. Al respecto, Abbas y Morbach (2005), mencionan que la educación sigue siendo la herramienta de prevención más poderosa en los países subdesarrollados y debería ser parte integral de los programas de prevención. Ésta debería ser simple, repetitiva y adaptarse culturalmente. Por otro lado, más de la mitad de las enfermeras no realiza la inspección del zapato y calcetines, resultado que se puede justificar por ser una práctica de actividad en atención primaria con pacientes ambulatorios. Dentro de este contexto, Brand (2005), manifiesta: "Quiten los zapatos y los calcetines al paciente y examínenle los pies, cada vez que vea a una persona con diabetes, revísele los pies". 
La gran mayoría de las enfermeras preguntan a sus pacientes si presentan cambios en la sensibilidad odolorenlas extremidades inferiores; al respecto, la investigación realizada por Pinilla, Barrera y Rodríguez (2010), señala que a la mayoría de los pacientes diabéticos no se les interrogó sobre alteraciones de la sensibilidad o dolor en el pie. Asimismo, más de la mitad de las enfermeras clasifica a sus pacientes en niveles bajo o alto riesgo e informan sobre su categoría de riesgo de desarrollar pie diabético, sin duda, se aprecia que las enfermeras continúan con las actividades de prevención, de acuerdo con, Brand (2005), "Quienes identifiquen como portadores de factores de riesgo deberían recibir una educación simple sobre cómo cuidar y conservar sus pies, si este consejo se aplicase en todo el mundo, tendría un enorme impacto sobre la reducción de la discapacidad y la muerte resultantes de la enfermedad de pie diabético." La mayoría de las enfermeras brinda técnicas de autocuidado del pie como: (a) cuidado de las uñas (b) lavado, secado y uso de cremas para el pie (c) revisión diaria del pie y (d) el tipo de zapatos recomendados para su actividad diaria, uso de medias y no caminar descalzo en casa u otros lugares. Al respecto, Gonzales (2008), refiere que la función de la enfermera consiste en ayudar al paciente a realizar acciones, por sí mismo, para conservar la salud y la vida, recuperarse o seguir el proceso de la enfermedad, afrontando las consecuencias y se debería realizar de modo complementario con la familia y la comunidad cuando está alterado el equilibrio.

En cuanto a la recomendación de dejar el hábito de fumar, la mayoría de las enfermeras exhorta a sus pacientes que abandonen la práctica de fumar, asimismo, instan a no dejar de asistir a su control en los centros hospitalarios para el seguimiento de la enfermedad, de igual modo, Gary y Clive (2005), señalan que el consumo de tabaco incrementa el riesgo de enfermedad cardiovascular y obstrucción de los vasos sanguíneos de las extremidades inferiores, altera la tolerancia a la glucosa en personas con diabetes, por lo tanto, dejar de fumar debería ser un componente importante del plan de control de las personas con diabetes.

Respecto, a las prácticas de autocuidado que realizan los pacientes diabéticos, casi la mitad presenta un nivel de autocuidado moderado de su pie, asimismo, en el estudio de González, Perdomo, Quintana, y Mosquera (2010), señala que hubo una mayor prevalencia del déficit de cuidados del pie en los pacientes el cual fue evidenciado por un corte inadecuado de uñas e higiene deficitaria.

La mayoría de pacientes no considera importante elegir el color de los calcetines al momento de comprarlos, hallazgo que se evidencia que no se cumple con las recomendaciones, de la misma manera en el estudio por Pinilla, Sánchez y Mejía (2011), encontró que la gran mayoría de los pacientes no tiene en consideración el color de las medias al momento de comprarlos.

Por otro lado, se aprecia que cerca de la mitad de los pacientes no revisan el interior de su calzado y más de la mitad no aplican cremas hidratantes. Es recomendable revisar los zapatos antes de ponérselos para retira piedrecitas, zonas arrugadas o abultamientos; asimismo, la hidratación es fundamental porque los pacientes diabéticos presentan resequedad de la piel se puede prevenirse y eliminarse, aplicándose crema hidratante en la planta, el dorso y el talón, pero no en los espacios interdigitales, al respecto, Trilla y Espluga (1997), señala que casi la mayoría de los pacientes no comprobaron el interior del zapato antes de calzarse, así concluyó señalando que la cantidad de autocuidado es muy baja en los pacientes diabéticos. Además, Montargil y Zanetti (2009), refieren que la mitad de los pacientes no hidrataba sus pies como parte de su autocuidado.

La mayor parte de paciente seca los espacios interdigitales del pie después de cada aseo siguiendo las recomendaciones para evitar la proliferación de gérmenes que pueden lesionar la piel y llegar a ser un factor que desencadene el pie diabético; asimismo, la mayoría de pacientes no camina descalzo en casa u otros lugares. Dentro de este contexto, en el estudio realizado por Pinilla, Sánchez, Mejía y Barrera (2011), señala que casi la mitad de los pacientes diabéticos seca su pie y menos de la tercera parte no camina descalzo en casa.

Un tercio de los pacientes no considera importante la temperatura del agua antes de asearse el pie. La higiene diaria de los pies es necesaria, antes de introducir el pie en el agua se debe comprobar la temperatura con el codo, para evitar quemaduras en pacientes con afectación neuropática. La mayoría evita el uso de objetos (compresa, botella, manta eléctrica) 
Actividades de prevención primaria del pie diabético y actividades de autocuidado en pacientes con diabetes tipo 2 en el Hospital II Clínica Geriátrica San Isidro Labrador, Essalud

para calentar su pie, al respecto, Gallardo, Puentes y Villalon (2010), señala que más de la mitad de los pacientes se aplica bolsas de agua caliente en los pies desconociendo el riesgo de lesión que les puede provocar, aumentando la probabilidad de padecer pie diabético y como consecuencia sufrir amputaciones de miembros inferiores.

Es interesante resaltar en este estudio que la mayoría de pacientes informo que sí comunicaría al médico si percibe algún problema en su pie, se aprecia de esta manera que los pacientes vienen supervisando sus pies como parte de su autocuidado; al respecto, McGill (2005), señala que la educación debería incluir instrucciones prácticas y sobre todo notificar a un profesional sanitario inmediatamente si se produce un corte, una ampolla o una rozadura en el pie. Sin embargo, casi la tercera parte de los pacientes informaron que continúan consumiendo alimentos fritos, este hallazgo revela la práctica de una alimentación inadecuada que provocará inestabilidad en el metabolismo de la glucosa y el aumento de peso con riesgo a enfermedades de origen cardiaco e instauración rápida de neuropatía diabética. Ante esta realidad, la Organización Mundial de la Salud (2013), señala: la ingesta de menos grasas saturadas y azucares, ha demostrado ser importante para mantener el peso adecuado y, por lo tanto, disminuir el riesgo de diabetes tipo2.

Más de la mitad de la muestra consume carne, pescado, huevos y más de dos carbohidratos al día. En la investigación de Pinilla, Sánchez y Mejía (2011), señalan que la tercera parte de los pacientes consumen más de dos fuentes de carbohidratos en las principales comidas del día. Un porcentaje menor que la mitad continúan agregando azúcar a sus alimentos y consumen productos que contengan endulzantes, aun los pacientes continúan ejerciendo hábitos de alimentación poco saludables que perjudican al metabolismo de la glucosa y la afectación de los nervios periféricos del pie. Al respecto, Seclén (2012), refiere que el alto consumo de alimentos procesados, carbohidratos, el exceso de harina, arroz y las gaseosas, han originado que la diabetes en la población aumente y la prueba más palpable es que la demanda de pacientes con diabetes es alta en consulta externa, hospitalización, emergencia y UCl.

Otro hallazgo que se encontró y debe ser mencionado, es que casi la mitad de los pacientes se encuentra con sobrepeso incluyendo ambos sexos y una minoría se encuentra con obesidad siendo el género femenino más afectado. Al respecto, Gonzales (2008), refiere: Se han identificado algunas condiciones de la persona con diabetes que aumentan la probabilidad de desarrollar una lesión en el pie: edad avanzada, sexo masculino, sobrepeso u obesidad, larga duración de la diabetes.

En este estudio, menos de la mitad de los varones presenta un nivel de autocuidado medio y más de la mitad de las mujeres mantiene un autocuidado medio, asimismo, un tercio de mujeres presentan un nivel de autocuidado alto. Al respecto, Cadena y Flores (2010), señalan que el nivel de autocuidado de la población por sexo reveló que tanto hombres como mujeres se encontraron con un nivel de autocuidado bueno, sin embargo, las mujeres obtuvieron mayor nivel de autocuidado. Asimismo, el estudio realizado por González, Perdomo, Quintana y Mosquera (2010), señala que más de la mitad de varones presentaron un nivel de cuidado deficiente, y solo la tercera parte de mujeres mantiene un deficiente autocuidado.

Declaración de financiamiento y de conflictos de interés:

El estudio fue financiado por los autores, quienes declaran no tener conflictos de interés.

\section{Correspondencia}

Rosa Mesares Damiano. Dirección: Madrid, España. Correo electrónico: rouz04@hotmail. com 


\section{REFERENCIAS BIBLIOGRÁFICAS}

Abbas Z. y Morbach S. (2015). Lesiones del pie en países en desarrollo: jurge educación! Revista Diabetes Voice. 50(17) Número especial. Recuperado desde: http://www.diabetesvoice. org.

Apelqvist y Ragnarson (2015). Calcular los costes del pie diabético. Revista Diabetes Voice. 50(11) Número especial. Recuperado desde: http://www. diabetesvoice.org

Asociación Latinoamericana de Diabetes (2010). Guías de diagnóstico, control y tratamiento de la diabetes mellitus tipo 2. Recuperado desde: http://www.alad-latinoamerica.org.

Asociación latinoamericana de diabetes (2006). Guías ALAD de diagnóstico control y tratamiento de la diabetes mellitus tipo 2. Recuperado desde:http// www.paho.org

Bakker Karel (2005). Boletín de la Federación Internacional de Diabetes, volumen 46, número 3.Recuperado desde: http://www.idf.org

Boletín Epidemiológico (2012). Vigilancia Epidemiológica de Diabetes en hospitales piloto.

Enero-diciembre 2012. (Lima). 21 (52): 877 - 879. Recuperado desde: http://www.dge.gob.pe.

Boada A. (2011). Lesiones cutáneas en el pie diabético, servicio de Dermatología, Hospital Universitari Germans Trias i Pujol, Universitat Autónoma de Barcelona, España.

Blanes J. (2011). Documento de consenso sobre el tratamiento de las infecciones en el pie del diabético, Rev Esp Quimioter; 24(4): 233-62.

Brand Paul (2005). El pie diabético: epidemiología, factores de riesgo y atención. Revista Diabetes Voice. 50(11) Número especial. Recuperado desde: http://www.diabetesvoice.org.

Cadena L. \& Flores G. (2010). Capacidades de autocuidado en pacientes con DM tipo 2. Tesis de licenciatura. Universidad Veracruzana. México.

Clarke Andrew (2005), Para preocuparse: patología no ulcerativa del pie. Revista Diabetes Voice. 50(22) Número especial. Recuperado desde: http://www.diabetesvoice.org.

Federación Internacional de la Diabetes (2011-2021). Plan mundial contra la diabetes. Recuperado de: http://www.idf.org
Federación Internacional de Diabetes (2013). Atlas de la diabetes $6^{a}$ edición. Recuperado desde:www. idf.org/diabetesatlas.

Figuera E. \& Duran V. (2011) Autocuidado en el adulto mayor con hiperqueratosis para prevenir el pie diabético. Revista electrónica de Portales Medico. Recuperado por: http://www.ulceras.net.

Fundación española del corazón (2012). Diabetes y riesgo cardiovascular, Recuperado de: http:// www.fundaciondelcorazon.com.

Gallardo P., Puentes M \& Villalon P. (2010) Percepción de riesgos en pacientes con diabetes mellitus durante el autocuidado de los pies. Revista cubana de Angiología y Cirugía Vascular. 11(1)66-75 Cuba.

Gallardo \& Zangronis (2008) Conocimientos y conductas de los pacientes con diabetes mellitus sobre el pie diabético. Revista cubana de medicina general integral 24(1), Habana.

García de los Ríos \& Aschner (2006). Guías ALAD de diagnóstico control y tratamiento de la Diabetes Mellitus Tipo 2. Recuperado por: http://www.aladlatinoamerica.org

González H., Perdomo E., Quintana L., Mosquera F. (2010). Estratificación de riesgo en pie diabético, gerokomos; 21 (4): 172-182 .Recuperado desde: http://scielo.isciii.es

Gómez \& Levy (2012) Seminarios de la Fundación Española de Reumatología. Pie diabético. 13(4):119-129. Recuperado desde: http://www. alad-latinoamerica.org

Instituto Nacional de Estadística (2013). Nota de Prensa No 063 - 29 de Abril 2013. Nueve de cada 100 adultos mayores fueron diagnosticados con diabetes. Recuperado desde: http://www.inei. gob.pe.

Jiménez \& Martínez (2007). Nivel de conocimientos del paciente diabético sobre la prevención del pie diabético. Revista Científica Electrónica de las Ciencia Medicas en Cienfuegos.5 (2). Cuba ISSN: 1727-897X.

Lafuente R. Cruz A. Batres S. Granados A. Castilla R. (2003), Guía de atención enfermera a personas con diabetes. $2^{a}$ Ed., Sevilla. España.

Martínez A., Pascual R. (2009). Valoración del riesgo de pie diabético en el paciente anciano en una consulta de enfermería. Revista Científica Gerokomos v.20 n.2 Madrid jun. 2009. 
Actividades de prevención primaria del pie diabético y actividades de autocuidado en pacientes con diabetes tipo 2 en el Hospital II Clínica Geriátrica San Isidro Labrador, Essalud

Ministerio de Salud (2012). Boletín Epidemiológico SE № 45. Recuperado desde: http: //www.limaeste. gob.pe

Montargil \& Zanetti (2009) Comportamiento y conocimiento: fundamentos para la prevención del pie diabético. Revista Acta aul Enferm; 22(1):17-23. Brasil

Monteagudo \& Segura (2010) Implementación de un programa de educación terapéutica para pacientes diabéticos hospitalizados que inician tratamiento con insulina. Revista Avances en Diabetología.26:281-5

Organización Mundial de la Salud (2013). Temas de salud: Diabetes. Recuperado desde: http://www. who.int/topics/diabetes_mellitus/es.

Pinilla E, Sánchez L. \& Mejía A. (2011), Actividades de prevención del pie diabético en pacientes de consulta externa de primer nivel. Revista Salud Pública. 13 (2): 262- 273, Bogotá.

Pinilla R., Barrera P, Rodríguez M., Rubio C, Devia D. (2010). Prevalencia de las actividades de prevención del pie diabético y de los factores de riesgo asociados en pacientes diabéticos hospitalizados en la clínica Universitaria Carlos lleras Restrepo, Bogotá. Recuperado desde: http://www.bdigital.unal.edu.co

Ramón \& Martínez C. (2005) Atención de enfermería en la prevención y cuidados del pie diabético, Prevención podológica en el pie diabético. Revista española de podología, España.

Rebaza Flores A. (2011). Diabetes Mellitus "Una Nueva Epidemia". Boletín Epidemiológico del Instituto Nacional de Rehabilitación №5. Recuperado desde: http://www.inr.gob.pe

Santa Biblia (1960) Versión Reina Valera. Éxodo 15:26

Seclén Santisteban S. (2012). Ocho de cada diez personas con diabetes mueren por infarto al Miocardio. Recuperado desde: http://www.minsa. gob.pe.

White, E. (1969). El ministerio de curación: Buenos Aires Editorial ACES.

Recibido: $16 / 02 / 17$

Aceptado: $20 / 08 / 17$ 